\title{
AN EXTENSION OF THE FUGLEDE-PUTNAM THEOREM TO SUBNORMAL OPERATORS USING A HILBERT-SCHMIDT NORM INEQUALITY
}

\author{
TAKAYUKI FURUTA
}

\begin{abstract}
We prove that if $A$ and $B^{*}$ are subnormal operators acting on a Hilbert space, then for every bounded linear operator $X$, the Hilbert-Schmidt norm of $A X-X B$ is greater than or equal to the Hilbert-Schmidt norm of $A^{*} X-X B^{*}$. In particular, $A X=X B$ implies $A^{*} X=X B^{*}$. In addition, if we assume $X$ is a Hilbert-Schmidt operator, we can relax the subnormality conditions to hyponormality and still retain the inequality.
\end{abstract}

1. In this paper an operator means a bounded linear operator on a separable infinite dimensional Hilbert space $H$. Let $B(H)$ and $C_{2}$ denote the class of all bounded linear operators acting on $H$ and the Hilbert-Schmidt class in $B(H)$, respectively. It is known that $C_{2}$ forms a two-sided ideal in the algebra $B(H)$ and $C_{2}$ is itself a Hilbert space for the inner product

$$
(X, Y)=\Sigma\left(X e_{j}, Y e_{j}\right)=\operatorname{Tr}\left(Y^{*} X\right)=\operatorname{Tr}\left(X Y^{*}\right)
$$

where $\left\{e_{j}\right\}$ is any orthonormal basis of $H$ and $\operatorname{Tr}()$ denotes the trace. In what follows, \|\|$_{2}$ denotes the Hilbert-Schmidt norm.

An operator $T$ is called subnormal if $T$ has a normal extension and hyponormal if $T^{*} T \geqslant T T^{*}$. The inclusion relation of these classes of nonnormal operators is as follows:

$$
\text { Normal } \varsubsetneqq \text { Subnormal } \varsubsetneqq \text { Hyponormal. }
$$

The above inclusions are all proper [5, Problem 160, p. 101].

THEOREM A [9]. If $A$ and $B$ are normal, then

$$
\|A X-X B\|_{2}=\left\|A^{*} X-X B^{*}\right\|_{2}
$$

for every $X \in B(H)$.

THeOREM B [3]. If $A$ and $B^{*}$ are subnormal operators and if $X$ is an operator such that $A X=X B$, then $A^{*} X=X B^{*}$.

In this paper we integrate Theorem A and Theorem B in order to prove a slightly stronger Theorem 1. Moreover in our Theorem 2 we have an extension of Weiss [8, Theorem 3] and Berberian [2, Theorem]. Finally we shall pose an open problem with respect to Theorem 1 .

Received by the editors July 3, 1979 and, in revised form, October 25, 1979 and February 15, 1980. 1980 Mathematics Subject Classification. Primary 47B10, 47B15, 47B20; Secondary 47A30.

Key words and phrases. Subnormal operator, hyponormal operator, Hilbert-Schmidt class. 
2.

THEOREM 1. If $A$ and $B^{*}$ are subnormal, then the following inequality holds:

$$
\|A X-X B\|_{2}>\left\|A^{*} X-X B^{*}\right\|_{2}
$$

for every $X \in B(H)$. The equality holds for every $X \in B(H)$ when $A$ and $B$ are both normal.

Proof. Since $A$ is subnormal, there exists a normal extension $\tilde{N}_{A}$ of $A$ on the Hilbert space $H \oplus H$ whose restriction to $H \oplus\{0\}$ is $A$ [4], that is, $\tilde{N}_{A}$ is given by

$$
\tilde{N}_{A}=\left(\begin{array}{cc}
A & A_{12} \\
0 & A_{22}
\end{array}\right)
$$

on $H \oplus H$. Also a normal extension $\tilde{N}_{B^{*}}$ of $B^{*}$ on $H \oplus H$ is given by

$$
\tilde{N}_{B^{*}}=\left(\begin{array}{cc}
B^{*} & B_{12} \\
0 & B_{22}
\end{array}\right)
$$

on $H \oplus H$. Put $\tilde{X}$ on $H \oplus H$ as follows:

$$
\tilde{X}=\left(\begin{array}{cc}
X & 0 \\
0 & 0
\end{array}\right)
$$

since $\tilde{N}_{B^{*}}^{*}$ is also normal, Theorem A easily implies

$$
\left\|\tilde{N}_{A} \tilde{X}-\tilde{X} \tilde{N}_{B^{*}}^{*}\right\|_{2}=\left\|\tilde{N}_{A}^{*} \tilde{X}-\tilde{X} \tilde{N}_{B^{*}}\right\|_{2}
$$

that is,

$$
\left\|\left(\begin{array}{cc}
A X-X B & 0 \\
0 & 0
\end{array}\right)\right\|_{2}=\left\|\left(\begin{array}{cc}
A^{*} X-X B^{*} & -X B_{12} \\
A_{12}^{*} X & 0
\end{array}\right)\right\|_{2}
$$

so that

$$
\|A X-X B\|_{2}^{2}=\left\|A^{*} X-X B^{*}\right\|_{2}^{2}+\left\|A_{12}^{*} X\right\|_{2}^{2}+\left\|X B_{12}\right\|_{2}^{2}
$$

The equation (1) yields

$$
\|A X-X B\|_{2}>\left\|A^{*} X-X B^{*}\right\|_{2}
$$

which is the desired norm inequality. When $A$ and $B$ are both normal, then $A_{12}=0$ and $B_{12}=0$ in (1), so that the equality holds in (*), so the proof is complete.

The following corollary follows by Theorem 1 .

COROLlary 1 [3]. If $A$ and $B^{*}$ are subnormal and $X$ is an operator such that $A X=X B$, then $A^{*} X=X B^{*}$.

Corollary 1 is some extension of the Fuglede-Putnam theorem [1], [5] and [7].

REMarK 1. As stated in the proof of the equality in Theorem $1,\left\|A_{12}^{*} X\right\|_{2}^{2}+$ $\left\|X B_{12}\right\|_{2}^{2}$ in (1) is considered as the perturbed term of the difference between normality and subnormality.

3. In this section, we relax the hypotheses on $A$ and $B^{*}$ in Theorem 1 to hyponormality and strengthen the hypothesis on $X$ to be in the Hilbert-Schmidt class. 
THEOREM 2. If $A$ and $B^{*}$ are hyponormal, then the following inequality holds:

$$
\|A X-X B\|_{2}>\left\|A^{*} X-X B^{*}\right\|_{2}
$$

for every $X$ in Hilbert-Schmidt class. The equality holds when $A$ and $B$ are both normal.

Proof. Define an operator $\mathcal{T}$ on $C_{2}$ as follows: $\mathcal{T} X=A X-X B$. Then, if we view $C_{2}$ as an underlying Hilbert space, then $\mathcal{T}^{*}$ exists and is easily verified to be given by $\mathcal{T}^{*} X=A^{*} X-X B^{*}$. Also

$$
\begin{aligned}
\left(\mathcal{T} * \mathcal{J}-\mathcal{T}^{*}\right) X= & A^{*}(A X-X B)-(A X-X B) B^{*} \\
& -\left\{A\left(A^{*} X-X B^{*}\right)-\left(A^{*} X-X B^{*}\right) B\right\} \\
= & \left(A^{*} A-A A^{*}\right) X+X\left(B B^{*}-B^{*} B\right) .
\end{aligned}
$$

Left and right multiplication acting on $C_{2}$ as the Hilbert space by a positive operator is itself a positive operator. Since $\mathcal{T}^{*} \mathcal{T}-\mathcal{T}^{*} \mathcal{T}^{*}$ is the sum of two positive operators by the hyponormality of $A$ and $B^{*}, \mathcal{T}$ is hyponormal. Therefore $\|\mathcal{T} X\|_{2}$ $>\|\mathcal{T} * X\|_{2}$ that is,

$$
\|A X-X B\|_{2} \geqslant\left\|A^{*} X-X B^{*}\right\|_{2} \text {. }
$$

The proof of equality follows by (2) and (3).

REMARK 2. Berberian [2, Theorem] shows that if $A$ and $B^{*}$ are hyponormal, then $A X=X B$ implies $A^{*} X=X B^{*}$ for an operator $X$ in Hilbert-Schmidt class and this is just the case of the equality for an operator $X$ in Theorem 2 . Weiss [8, Theorem 3] shows the case of the equality in Theorem 2 when $A=B$ is normal, by a different method.

REMARK 3. It is of interest to remark that Theorem 1, Theorem 2 and Corollary 1 do not involve symmetric hypotheses on $A$ and $B$, but rather on $A$ and $B^{*}$. In view of this point, it is natural and reasonable in Theorem $A$ to interpret the hypothesis of normality of $A$ and $B$ as that of normality of $A$ and $B^{*}$.

Open problem. Can the subnormality be relaxed by the hyponormality in Theorem 1 ? This is an open problem.

We would like to express our thanks to the referee for his kind advice.

\section{REFERENCES}

1. S. K. Berberian, Note on a theorem of Fuglede and Putnam, Proc. Amer. Math. Soc. 10 (1959), 175-182.

2. , Extensions of a theorem of Fuglede and Putnam, Proc. Amer. Math. Soc. 71 (1978), 113-114.

3. T. Furuta, Relaxation of normality in the Fuglede-Putnam theorem, Proc. Amer. Math. Soc. 77 (1979), 324-328.

4. P. R. Halmos, Shifts on Hilbert spaces, J. Reine Angew. Math. 208 (1961), 102-112.

5. __ A Hilbert space problem book, Van Nostrand, Princeton, N.J., 1967.

6. C. R. Putnam, On normal operators in Hilbert space, Amer. J. Math. 73 (1951), 357-362.

7. M. Rosenblum, On a theorem of Fuglede and Putnam, J. London Math. Soc. 33 (1958), 376-377.

8. G. Weiss, The Fuglede commutativity theorem modulo operator ideals (to appear).

9. , Fuglede's commutativity theorem modulo the Hilbert-Schmidt class and generating functions for matrix operators. II (to appear).

Departiment of Mathematics, Faculty of Science, Hirosaki University, Bunkyo-cho 3, HirosAkI, 036 AOMORI, JAPAN 